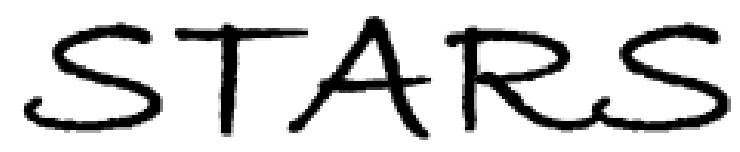

University of Central Florida

STARS

$1-1-2012$

\title{
Efficiency determination of an electrostatic lunar dust collector by discrete element method
}

Nima Afshar-Mohajer

Chang-Yu Wu

Nicoleta Sorloaica-Hickman

University of Central Florida

Find similar works at: https://stars.library.ucf.edu/facultybib2010

University of Central Florida Libraries http://library.ucf.edu

This Article is brought to you for free and open access by the Faculty Bibliography at STARS. It has been accepted for inclusion in Faculty Bibliography 2010 s by an authorized administrator of STARS. For more information, please contact STARS@ucf.edu.

\section{Recommended Citation}

Afshar-Mohajer, Nima; Wu, Chang-Yu; and Sorloaica-Hickman, Nicoleta, "Efficiency determination of an electrostatic lunar dust collector by discrete element method" (2012). Faculty Bibliography 2010s. 2193. https://stars.library.ucf.edu/facultybib2010/2193

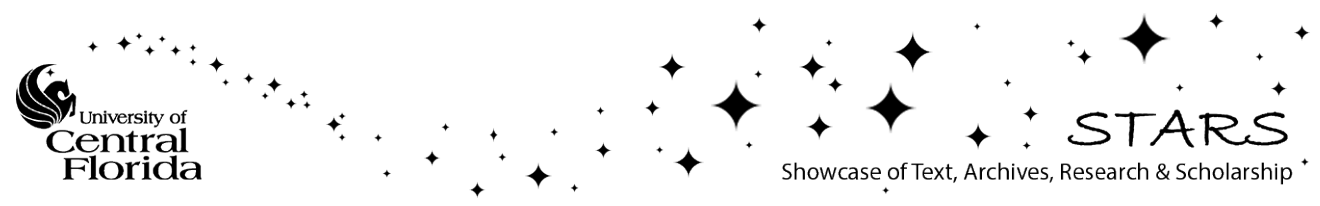




\section{Efficiency determination of an electrostatic lunar dust collector by discrete element method}

Cite as: J. Appl. Phys. 112, 023305 (2012); https://doi.org/10.1063/1.4739734

Submitted: 30 April 2012 . Accepted: 27 June 2012 . Published Online: 31 July 2012

Nima Afshar-Mohajer, Chang-Yu Wu, and Nicoleta Sorloaica-Hickman

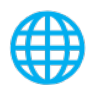

\section{ARTICLES YOU MAY BE INTERESTED IN}

Electrostatic Dust Control on Planetary Surfaces

AIP Conference Proceedings 880, 400 (2007); https://doi.org/10.1063/1.2437479

\section{Applied Physics Reviews} Now accepting original research

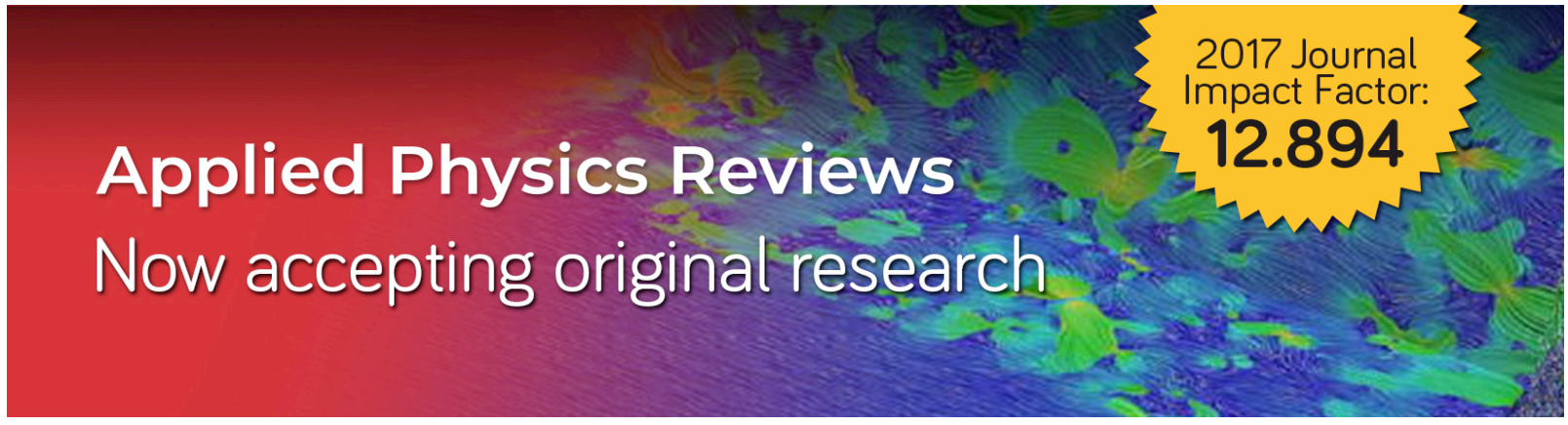




\title{
Efficiency determination of an electrostatic lunar dust collector by discrete element method
}

\author{
Nima Afshar-Mohajer, ${ }^{1}$ Chang-Yu Wu, ${ }^{1, a)}$ and Nicoleta Sorloaica-Hickman ${ }^{2}$ \\ ${ }^{1}$ Department of Environmental Engineering Sciences, University of Florida, Gainesville, Florida 3261 1, USA \\ ${ }^{2}$ Florida Solar Energy Center, University of Central Florida, Cocoa, Florida 32922, USA
}

(Received 30 April 2012; accepted 27 June 2012; published online 31 July 2012)

\begin{abstract}
Lunar grains become charged by the sun's radiation in the tenuous atmosphere of the moon. This leads to lunar dust levitation and particle deposition which often create serious problems in the costly system deployed in lunar exploration. In this study, an electrostatic lunar dust collector (ELDC) is proposed to address the issue and the discrete element method (DEM) is used to investigate the effects of electrical particle-particle interactions, non-uniformity of the electrostatic field, and characteristics of the ELDC. The simulations on 20- $\mu \mathrm{m}$-sized lunar particles reveal the electrical particle-particle interactions of the dust particles within the ELDC plates require 29\% higher electrostatic field strength than that without the interactions for $100 \%$ collection efficiency. For the given ELDC geometry, consideration of non-uniformity of the electrostatic field along with electrical interactions between particles on the same ELDC geometry leads to a higher requirement of $\sim 3.5 \mathrm{kV} / \mathrm{m}$ to ensure $100 \%$ particle collection. Notably, such an electrostatic field is about $10^{3}$ times less than required for electrodynamic self-cleaning methods. Finally, it is shown for a "halfsize" system that the DEM model predicts greater collection efficiency than the Eulerian-based model at all voltages less than required for $100 \%$ efficiency. Halving the ELDC dimensions boosts the particle concentration inside the ELDC, as well as the resulting field strength for a given voltage. Though a lunar photovoltaic system was the subject, the results of this study are useful for evaluation of any system for collecting charged particles in other high vacuum environment using an electrostatic field. @ 2012 American Institute of Physics. [http://dx.doi.org/10.1063/1.4739734]
\end{abstract}

\section{INTRODUCTION}

Clouds of levitated dust particles in the lunar environment disturbed National Aeronautics and Space Administration's (NASA) exploratory missions during the Apollo era. ${ }^{1-4}$ Abrasive and levitated lunar particles caused significant problems for astronauts, extravehicular activities, spacecraft, and investigative equipment. ${ }^{2,5}$ As a result, NASA has established active lunar dust mitigation programs to develop technologies for mitigating and controlling lunar dust for future explorations in the lunar environment.

Meteorite impacts, human activities, and movement of lunar rovers all contribute to kinetic levitation of the grains. ${ }^{6}$ However, electrically driven dust levitation due to charge accumulation on the lunar surface is well known to be the reason for most lunar dust levitation., ${ }^{2,5}$ With practically no atmospheric shielding, ${ }^{1,3,7}$ the lunar surface is exposed to both intense solar radiation (UV, $\mathrm{x}$-ray, and cosmic rays) and solar winds (high velocity charged particles) which charge particles on the lunar surface. ${ }^{3}$ On the dayside of the moon, the sun's electromagnetic radiation make lunar grains positively charged, while impingement of low energy electrons in the solar wind dominates on the nightside of the moon, making lunar grains negatively charged. ${ }^{3,4,8-11}$

The like-charged dust particles on each side of the moon then repel each other, overcoming gravitational and cohesive forces. Eventually, the process of charge accumulation inside

\footnotetext{
a) Author to whom correspondence should be addressed. Electronic mail: cywu@ufl.edu. Tel.: (352)392-0845. Fax: (352)392-3076.
}

the Debye length leads to lifting the particles from the surface. ${ }^{2,12,13}$ The resulting electric field on the lunar surface forms a sheath region with non-uniform thickness (from less than $10 \mathrm{~cm}$ to several tens of meters) around the moon. ${ }^{14}$ Relatively smaller and lighter lunar particles are forced up outside the region to the point where the electric field is screened out and no longer acts on the particles. ${ }^{10}$ Thus, the majority of levitated particles fall back toward the lunar surface and deposit on the exposed surfaces with time. ${ }^{12}$ Dust movement is further agitated by the alternating day to night cycle of any region of the lunar surface, such that the sheath region alternates between a positive and negative charge characteristic.

Dust deposition on surfaces of investigative hardware installed on the lunar surface deteriorates the performance of most mechanical systems. This leads to false instrument measurements, thermal control problems, ${ }^{5}$ abrasion of space suits and dust penetration inside the cabins, ${ }^{15}$ dust stickiness to lunar rovers, ${ }^{3}$ damage in optical surfaces, ${ }^{4}$ and ultimately the need for costly replacement of exposed surfaces. Obscurity of solar photovoltaic devices is one of the most important problems from lunar dust deposition, as it significantly reduces the solar panel efficiency. ${ }^{16}$

Several methods have been surveyed to protect optical or photovoltaic surfaces from the lunar dust deposition threat, but the constraints of the lunar environment make them either inefficient or infeasible. ${ }^{7}$ In hard vacuum environments, application of mechanical removal methods such as brushing and wiping are impractical as they are inconvenient for astronauts 
and difficult to implement robotically. Moreover, human activities close to the surfaces deposit even more particles on them. Gases, liquids, foams, and gels expand in all directions, evaporate or freeze in the lunar environment, and leave residues with unknown effects on lunar atmosphere. ${ }^{17}$ Since there is no drag force on particles in the lunar atmosphere and the lunar magnetic field is negligible (1 to $10 \mathrm{nT}),{ }^{18}$ applying an electric field would be the best approach for controlling the naturally charged lunar dust.

On this basis, an electrodynamic transparent dust shield was proposed by Biris et al. ${ }^{16}$ as a self-cleaning method to protect solar cells in the lunar environment. The system uses embedded parallel wires on a transparent polymer film while an AC signal is applied. The results from this study demonstrated that the performance of electrodynamic dust shield is a function of amplitude, frequency, and shape of the embedded electrodes. Because this technology treats already settled particles with AC signals, significant electric potential should be provided to overcome surface forces and remove the particles. ${ }^{16}$

In the present study, the collection efficiency of an electrostatic lunar dust collector (ELDC) ${ }^{17}$ was evaluated as a mitigating technology to protect solar panel surfaces. The adequateness of a relatively low electrostatic field between a series of conducting plates had been demonstrated previously $\left(\mathrm{E}=20 \mathrm{kV} \mathrm{m}^{-1}\right.$ for the worst case scenario to collect all the particles smaller than $\mathrm{d}_{\mathrm{p}}=140 \mu \mathrm{m}$ ) based on an Eulerian model with simplifying assumptions. ${ }^{17}$ However, the simple Eulerian model could not consider electrical particle-particle interactions and fringe effects due to limiting aspect ratio of the ELDC, as those require tracking the particle positions with respect to other particles and ELDC geometry. Since the electrical particle-particle interactions and non-uniformity of charge distribution on the ELDC plates may affect the collection efficiency of the proposed system, this study was embarked to address more accurate determination of the ELDC collection efficiency based on the discrete element method (DEM). The proposed system is capable of efficiently protecting solar panel surfaces from the settling charged lunar dust using a small fraction of electric power provided from the solar panel itself.

\section{METHOD}

\section{A. ELDC configuration}

The ELDC consists of a grid layer of rectangular and parallel thin plates in front of the surface to be protected. The ELDC plates are conducting, and hard vacuum condition between the plates acts as an insulator to practically form a capacitor. Providing electrical potential difference between each pair of plates, an electrostatic field is created to collect falling dust particles before surface impact. Since the solar panels can store energy in the lunar day for all the equipment, the provision of the required voltage between the plates can be easily maintained, and the collection of particles on the plates does not relax and dump down on the PV cell.

The collection efficiency of the ELDC depends on its orientation. The most conservative efficiency occurs when the ELDC plates are normal to the lunar surface. ${ }^{17}$ Under this situation, gravitational force on falling particles accelerates them toward the surface. Since there is no drag force on particles in the hard vacuum of the lunar environment, the electrostatic field from the plates provides an effective means to avoid surface impact (see Fig. 1).

Lunar dust particles are falling down from their maximum height of levitation with a zero initial velocity as free-fall objects that accelerate due to the lunar gravity. ${ }^{2}$ Procedures to calculate the particles' velocities at the entrance of the ELDC are explained in Sec. II B. Since there is no drag force acting on particles in lunar atmosphere, all the particles fall at the same acceleration $\left(\mathrm{g}_{1}=1.62 \mathrm{~m} / \mathrm{s}^{2}\right)$. However, particle size is important in determining deflection of the falling particle toward the collecting ELDC plate, and the accumulated surface charge on a particle increases linearly with particle diameter. ${ }^{8,19,20}$ On the other hand, vertical acceleration due to lunar gravity is proportional to $\mathrm{d}_{\mathrm{p}}{ }^{-3}$, making the larger particles harder to collect.

\section{B. Properties of lunar dust particles}

For simplicity of the later numerical computations, it is assumed that particles enter the ELDC with identical size, shape, and density. This means appropriate and representative values for particle size, particle density, and particle shape should be taken. Unlike terrestrial dust, lunar dust is exposed to meteorite impacts. These hypervelocity impacts result in shape irregularity and fragmentation of lunar grains in a wide range of size distribution (from nanometer to centimeter). ${ }^{3,7}$ Between $10 \%$ and $20 \%$ of lunar grains by number are smaller than $20 \mu \mathrm{m} .{ }^{21}$ Over $95 \%$ and over $50 \%$ are finer than $1 \mathrm{~mm}$ and $60 \mu \mathrm{m}$, respectively. ${ }^{22}$ The average value for median size has been reported as $70 \mu \mathrm{m} .{ }^{15}$ Lunar dust density ranges from 2300 to $3100 \mathrm{~kg} / \mathrm{m}^{3}{ }^{15}$ As a conservative

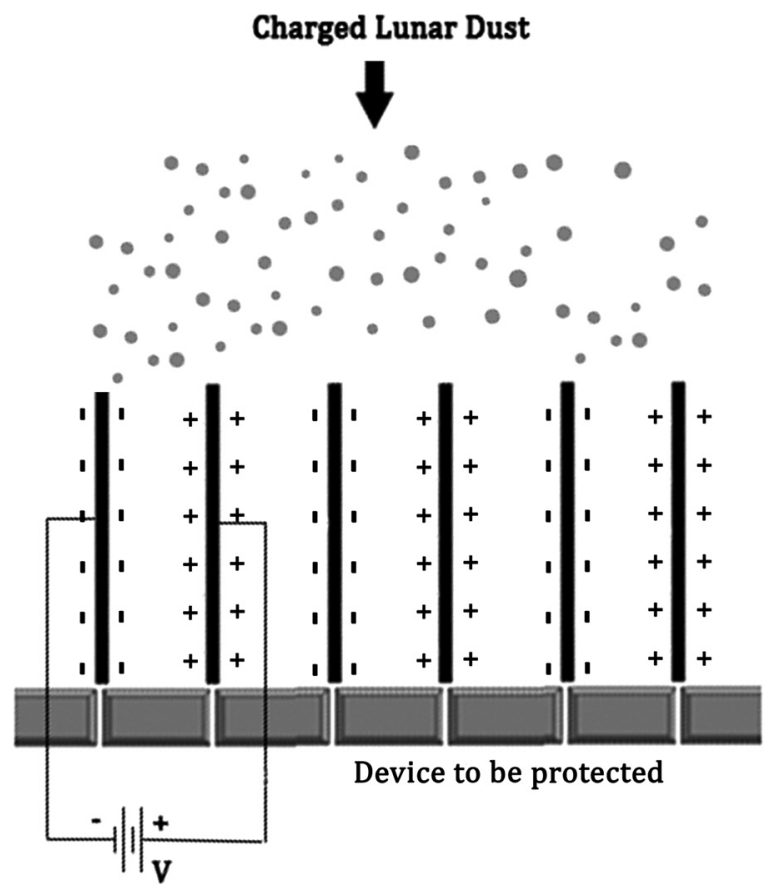

FIG. 1. Schematic of the ELDC configuration to protect optical/photovoltaic surfaces at the worst case scenario. 
estimate for particle collection efficiency, the upper limit value for lunar dust density $\left(\rho_{\mathrm{p}}=3100 \mathrm{~kg} / \mathrm{m}^{3}\right)$ was chosen. With no knowledge of the shape distribution, all the particles were modeled as spherical.

In general, the size of the particles influences levitation height, particle charge, and particle initial velocity at the entrance of the ELDC. Thus, particle size is an independent variable for several involved dependent parameters. Although relatively larger particles are more difficult to be collected, they might not have enough levitation height to threaten an exposed surface. Hence, the largest particle size which can be adequately levitated from the lunar surface is determined. According to Stubbs et al., ${ }^{2}$ small lunar dust leaves the sheath region outside of which only lunar gravity acts on particle and it follows a nearparabolic trajectory to fall back toward the lunar surface. The maximum levitation height is a function of particle size and surface potential of the grain ${ }^{2}$

$$
\mathrm{Z}_{\max }=\frac{12 \varepsilon_{0} \phi_{\mathrm{s}}^{2}}{\rho_{\mathrm{p}} \mathrm{g}_{1} \mathrm{~d}_{\mathrm{p}}^{2}}
$$

where $\varepsilon_{0}$ and $\mathrm{g}_{1}$ are vacuum space permittivity $(8.854 \times$ $\left.10^{-12} \mathrm{~F} / \mathrm{m}\right)$ and lunar gravitational acceleration $\left(\sim 1.62 \mathrm{~m} / \mathrm{s}^{2}\right)$.

Although both in situ measurements and theoretical equations have been attempted for lunar surface potential $\left(\phi_{\mathrm{s}}\right)$ estimation, its value has not yet been well characterized. The surface potential ranges from a few volts to a few hundred volts, and it varies considerably with both time and space. In this study, $\phi_{\mathrm{s}}=100 \mathrm{~V}$ was taken as the representative value for the surface potential. Assuming $50 \mathrm{~cm}$ is the minimum levitation height for a particle to be considered a threat for an exposed surface, Eq. (1) gives the maximum size of the lunar particle approximately $d_{p}=20 \mu \mathrm{m}$. Equations (2) and (3) were used to calculate the particle charge and initial velocity at the entrance of the ELDC (when $\mathrm{d}_{\mathrm{p}}=20 \mu \mathrm{m}$ and $\left.\phi_{\mathrm{s}}= \pm 100 \mathrm{~V}\right)^{17,23}$

$$
\begin{aligned}
& \mathrm{q}_{\mathrm{p}}=2 \pi \varepsilon_{0} \mathrm{~d}_{\mathrm{p}} \phi_{\mathrm{s}}, \\
& v_{0 \mathrm{p}}=\frac{2 \phi_{\mathrm{s}}}{\mathrm{d}_{\mathrm{p}}} \sqrt{\frac{6 \varepsilon_{0}}{\rho_{p}} .}
\end{aligned}
$$

The polarity of all the particles on a given side of the moon is the same. Since all dust particles approaching the ELDC are positive (dayside) or negative (nightside), there would be repelling actions among the particles. This means in addition to gravitational force $\left(\mathrm{F}_{\mathrm{g}}\right)$ and provided electrostatic force $\left(\mathrm{F}_{\mathrm{e}}\right)$ from the collecting ELDC plate, there would be repelling Coulomb forces on any target particle from all the other particles.

\section{Discrete element method}

To address the mechanical contacts and electrical interactions of the falling particles, a Lagrangian approach is required to track particles individually at each time step. In this study, the DEM which has been widely used in particulate systems was implemented for tracking the particles. ${ }^{23}$
The basic idea of DEM modeling is placement of the initial set of particles followed by consideration of all the acting forces on any individual particle. Then, Newton's second law and kinematic equation conclude updated positions of the particles for the next time step. ${ }^{24}$ EDEM 2.3.1 developed by DEM Solutions, Inc., which has incorporated electrostatic forces into DEM computations, was adopted for this study. Flexibility in creating different geometries, ease of conducting sensitivity analyses, and capability of designing more complicated particle characteristics are other advantages of the software.

Contact detection is the most time-consuming part in DEM modeling of a particulate system (for a system made of $\mathrm{n}$ particles, on the order of $\mathrm{n}^{2}$ detection checks are required at each time step). ${ }^{24}$ Moreover, there are electrostatic particle-particle interactions among the charged particles. The EDEM uses a Cartesian grid on the computational domain to limit particle contact detection to the number of particles located in the same or adjacent grid cells. A soft contact force model (Hertz-Mindlin with no particle slip) was used for the mechanical contacts. This model is based on frictional elasticity of a spherical particle in contact with wall or other particles (Hertz model for the normal direction and Mindlin model for the tangential direction). ${ }^{25}$ As for electrostatic interactions, a screening radius around all particles is defined. For any target particle, only those particles inside its screening distance are involved in calculations of electrical interactions. ${ }^{23}$ The electrostatic screening distance in EDEM is practically the Debye length of the particles, $\lambda_{\text {Dp. }}$. The following equation is the suggested Debye length in vacuum: ${ }^{24}$

$$
\lambda_{\mathrm{Dp}}=\frac{1}{\mathrm{q}_{\mathrm{e}} \sqrt{\left(\frac{1}{\varepsilon_{0} \mathrm{~K}_{\mathrm{B}} \mathrm{T}} \sum_{\mathrm{i}} \mathrm{n}_{\mathrm{i}} \mathrm{z}_{\mathrm{i}}^{2}\right)}},
$$

where $\mathrm{q}_{\mathrm{e}}$ is unit charge of an electron $\left(1.602 \times 10^{-19} \mathrm{C}\right), \mathrm{K}_{\mathrm{B}}$ is Boltzmann's constant $\left(1.38 \times 10^{-23} \mathrm{~J} / \mathrm{K}\right)$, T is temperature in Kelvin, $n_{i}$ is number of i-type particles of charge $z_{i}$. Thus, the model considers a circle around each particle with $\lambda_{\text {Dp }}$ as radius and calculates the electrostatic potential $\left(\mathrm{U}_{\mathrm{e}}\right)$ between the center particle and each particle inside the circle

$$
\mathrm{U}_{\mathrm{e}}=\frac{\mathrm{q}_{\mathrm{p}} \mathrm{q}_{\mathrm{i}}}{4 \pi \varepsilon_{0} \mathrm{r}_{\mathrm{pi}}} \mathrm{e}^{\frac{-\mathrm{r} \mathrm{pi}}{\lambda_{\mathrm{Dp}}}}
$$

where $q_{i}$ is charge of particle $i$ inside the screening distance and $r_{p i}$ is distance between the center particle and particle $i$. The resultant Coulomb force from all the included particles is derived from electrostatic potential as shown in Eq. (6)

$$
\mathrm{F}_{\mathrm{c}}=-\sum_{\mathrm{i}}\left(\frac{\mathrm{d} \mathrm{U}_{\mathrm{e}}}{\mathrm{dr}_{p i}}\right)=-\sum_{\mathrm{i}}\left(\frac{\mathrm{q}_{\mathrm{p}} \mathrm{q}_{\mathrm{i}}}{4 \pi \varepsilon_{0}}\left(\frac{1}{\mathrm{r}_{\mathrm{pi}} \lambda_{\mathrm{Dp}}}+\frac{1}{\mathrm{r}_{\mathrm{pi}}{ }^{2}}\right) \mathrm{e}^{\frac{-\mathrm{r}_{\mathrm{pi}}}{\lambda_{\mathrm{Dp}}}}\right),
$$

Time step is the last factor influencing the accuracy and total run time of simulations. The adopted time step is the largest time in which all the above mentioned calculations can be performed accurately. The EDEM guideline suggests 
$40 \%$ to $60 \%$ of the Rayleigh time step to be taken as the time step of calculations. The Rayleigh time step, $t_{R}$, is the time taken for a shear wave to propagate through a solid particle, and it is defined as ${ }^{26}$

$$
t_{R}=\frac{\pi d_{p}}{\left(0.3262 v_{p}+1.7532\right)} \sqrt{\frac{\rho_{p}}{G_{p}}},
$$

where $\mathrm{G}_{\mathrm{p}}$ and $v_{\mathrm{p}}$ are particle shear modulus and particle Poisson's ratio, respectively. Lunar dust is mostly composed of glassy materials. ${ }^{27}$ So, these values for lunar dust were assumed to be analogous to glass, and $40 \%$ of the calculated $t_{R}$ was considered as the time step.

\section{Non-uniform charge distribution on ELDC plates}

The assumption of uniformity of the created electrostatic field is valid for the case if the spacing between the plates, $\mathrm{D}$, is fairly small compared to the length or width of the plates, $\mathrm{L}$ or $\mathrm{W} .{ }^{28}$ Providing a high L/D ratio in ELDC geometry may block optical or photovoltaic surfaces from the sun light. If the solar panel is not maintained normal to the sun by a tracking mechanism, extra spacing must be provided between the ELDC plates, so as to not shade portions of the active solar cells from oblique radiation. Such shading is of great concern in solar panels, since the performance deteriorates significantly with a small reduction in the absorbed sun beams. The limited ratio of $\mathrm{L} / \mathrm{D}=2$ in the above mentioned modeling is smaller than the recommended value for uniform electrostatic field validity in parallel plate capacitors. ${ }^{29}$ So, the second section of DEM modeling was designed to study the influence of the non-uniform charge distribution on ELDC plates and influence of charge accumulation at the edges (so-called fringe effect) on the generated electrostatic field and collection efficiency of the ELDC.

The charge distribution on the ELDC plates is denser at the edges. To create an accurate non-uniform electrostatic field between the plates, charge distribution on the ELDC plates was calculated. Each ELDC plate was divided equally into $\mathrm{n}$ pieces of squared subsections. The electrical potential of each subsection was derived from the integral solution of Poisson's equation with two assumptions: the surface charge is constant within each subsection, and electrical potential on all the subsections of a plate is equal and is a constant $\mathrm{t}^{30,31}$

$$
\mathrm{V}_{\mathrm{i}}=\int_{\mathrm{s}} \frac{\rho_{\mathrm{S}} \mathrm{ds}}{4 \pi \varepsilon_{0} \mathrm{R}}
$$

where for $\mathrm{i}=1,2, \ldots, \mathrm{n}$ on positively charged plate, $\mathrm{V}_{\mathrm{i}}$ (electrical potential of ith subsection) is equal to $\Delta \mathrm{V} / 2(\Delta \mathrm{V}$ to be the provided electrical potential between the ELDC plates), and for $\mathrm{i}=\mathrm{n}+1, \mathrm{n}+2, \ldots, 2 \mathrm{n}$ on negatively charged plate, $\mathrm{V}_{\mathrm{i}}$ is equal to $-\Delta \mathrm{V} / 2 . \mathrm{R}$ is position vector between any two points on the ELDC plates, $\rho_{\mathrm{s}}$ is surface charge density and $\mathrm{s}$ refers to area of each plate. Since each plate was discretized into n subsections, Eq. (8) was estimated using Eq. (9) where electrical potential on ith subsection is the product of charge interactions with the other subsections on the same plate and subsections on the opposite plate

$$
\begin{gathered}
\mathrm{V}_{\mathrm{i}} \cong \sum_{\mathrm{j}=1}^{2 \mathrm{n}} \rho_{\mathrm{j}} \frac{1}{4 \pi \varepsilon_{0}} \int_{\Delta \mathrm{s}_{\mathrm{i}}} \frac{\mathrm{ds}}{\mathrm{R}_{\mathrm{ij}}}=\sum_{\mathrm{j}=1}^{2 \mathrm{n}} \rho_{\mathrm{j}} \mathrm{A}_{\mathrm{ij}}, \\
\mathrm{A}_{\mathrm{ij}}=\frac{1}{4 \pi \varepsilon_{0}} \int_{\mathrm{y}=\mathrm{y}_{1}}^{\mathrm{y}=\mathrm{y}_{2}} \int_{\mathrm{x}=\mathrm{x}_{1}}^{\mathrm{x}=\mathrm{x}_{2}} \frac{\mathrm{dx} \mathrm{dy}}{\mathrm{R}_{\mathrm{ij}}}
\end{gathered}
$$

where $\rho_{\mathrm{j}}$ is the surface charge density of the jth subsection, $\Delta s_{i}$ is the area of ith subsection, $R_{i j}$ is the distance between ith subsection and jth subsection, and $A_{i j}$ is a $2 n \times 2 n$ matrix defined for the sake of convenience. As the subsections are squared, $d x=d y=\Delta l$. So, the matrix $A_{i j}$ for $i \neq j$ and $i=j$ cases is rewritten as

$$
\begin{gathered}
\mathrm{A}_{\mathrm{ij}}=\frac{(\Delta \mathrm{l})^{2}}{4 \pi \varepsilon_{0} \mathrm{R}_{\mathrm{ij}}} \quad(\text { for } \mathrm{i} \neq \mathrm{j}), \\
\mathrm{A}_{\mathrm{ij}}=\frac{\Delta \mathrm{l}}{\pi \varepsilon_{0}}(\ln (1+\sqrt{2}) \quad(\text { for } \mathrm{i}=\mathrm{j}) .
\end{gathered}
$$

Equations (8)-(12) relate electrical potential of a subsection $\left(\mathrm{V}_{\mathrm{i}}=\Delta \mathrm{V} / 2\right)$ to its surface charge. This set of equations was solved numerically using MATLAB 7.10.0 codes for different numbers of subsections to obtain $\rho_{\mathrm{i}}$ and total charge on the plate, Q. The capacitance, C, of the ELDC which is defined as ratio of total charge on a plate over the provided electrical potential, $\Delta \mathrm{V}$, was used to determine the appropriate number of subsections. So, the capacitance of the ELDC was plotted as a function of the number of subsections, and since the graph shows an asymptote with an increase in $\mathrm{n}$, the minimum number of subsections providing the observed asymptote was opted as $\mathrm{n}$ in computations.

\section{E. Simulation conditions}

On this basis, the numerical simulations were divided into three sections. In section 1, the ELDC geometry was adopted based on the dimensions of commercial photovoltaic cells on solar panel surfaces, i.e., $10 \mathrm{~cm} \times 10 \mathrm{~cm}$ squared plates which were $5 \mathrm{~cm}$ away from each other. It was also assumed the electrostatic field between the parallel plates is constant with time and everywhere between the plates (Fig. 2). In section 2, the ELDC geometry was kept the same but the effect of nonuniformity in charge distribution on the plates (fringe effect) on ELDC collection efficiency was addressed. Finally, in the third section, a half-size ELDC geometry (halving L, W, and $\mathrm{D}$ so the same $\mathrm{L} / \mathrm{D}$ and $\mathrm{W} / \mathrm{D}$ ratios) was examined to survey the influence of ELDC dimensions combined with the fringe effect on the collection efficiency.

Initially, 100 spherical particles $\left(\mathrm{d}_{\mathrm{p}}=20 \mu \mathrm{m}, \rho_{\mathrm{p}}\right.$ $=3100 \mathrm{~kg} / \mathrm{m}^{3}$, and $\phi_{\mathrm{s}}=100 \mathrm{~V}$ ) uniformly positioned at the entrance of ELDC (at $\mathrm{y}=0$ ) to cover the spacing between plates were modeled (i.e., the distance between two particles was $0.495 \mathrm{~mm}$ in sections 1 and 2 , and it was $0.2475 \mathrm{~mm}$ in section 3 of the simulations). Charge and initial velocity of the particles were assigned according to Eqs. (2) and (3). Before starting the main sets of simulations, a pre-simulation was conducted to validate the model with one single falling down particle with aforementioned characteristics (when there is no particle-particle interaction) at different initial 


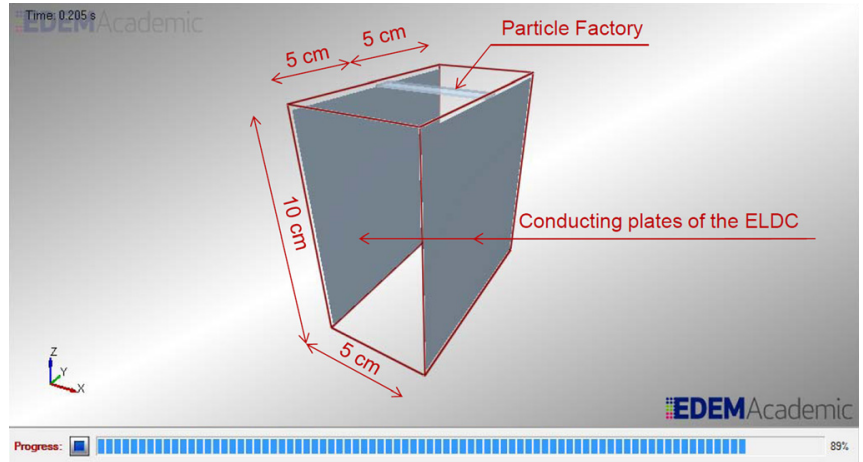

FIG. 2. The ELDC geometry and particle placement in sections 1 and 2 (100 lunar dust particles at the middle initially forming a line inside the particle factory; $\mathrm{L}=\mathrm{W}=10 \mathrm{~cm}$ and $\mathrm{D}=5 \mathrm{~cm}$ ).

positions at the ELDC entrance. The final position of the particle at each simulation was compared with the predicted result from formerly derived results of an Eulerian model. ${ }^{17}$ The identical results from both methods verified the validity of the DEM model for the three-sectioned simulations of this study. All the simulations were surveyed at $\Delta \mathrm{V}=25,50,75$, and $100 \mathrm{~V}$.

Then the ELDC plates in the DEM model were divided into subsections and the calculated surface charges were assigned as point charges at the center of each subsection. The DEM simulations were restarted at this non-uniform electrostatic field. Since the surface charge distribution on ELDC plates was no longer uniform, the line of 100 particles at the ELDC entrance was located at 5 different positions in the $\mathrm{y}$ direction $(\mathrm{y}=-4,-2,0,+2,+4 \mathrm{~cm})$ to cover the entire $10-\mathrm{cm}$ width. So, falling particles were exposed to different areas of the ELDC plates with different surface charges. Then, the obtained collection efficiencies at different $y$ were averaged to get the representative collection efficiency.

\section{RESULTS AND DISCUSSION}

\section{A. Effect of electrical particle-particle interactions}

In the first set of simulations, charge distribution on ELDC plates was assumed ideally uniform. This means horizontal acceleration due to the external electrostatic field on falling particle is a constant value (as well as vertical acceleration which is simply gravitational acceleration). The Coulomb forces among 100 like-charged particles complicate the model. Since the main focus of these simulations was electrostatic interactions, the cell grid size for mechanical contacts was not limiting. The effect of cell grid size on 100 incoming particles was surveyed and no mechanical contact was recorded for this number of particles in the applied geometry (the cell grid size was fixed to $50 \mathrm{~d}_{\mathrm{p}}$ in the main simulations).

The particle concentration and electrical screening distance around the particles are two key parameters in setting up the DEM model. Referring to Eq. (4), the required electrical screening distance for $20-\mu \mathrm{m}$-sized particles is a few meters which is larger than the ELDC geometry. As the screening distance significantly influences the simulation run time, a sensitivity analysis was conducted on particle's Debye length. As displayed in Fig. 3, obtained collection efficiencies at $\lambda_{\text {Dp }}$ greater than $2.5 \mathrm{~mm}$ are fairly constant. This means at this concentration of particles taking any value larger than $2.5 \mathrm{~mm}$ as the screening distance does not matter. On the other hand, the electrical particle-particle interaction is dependent on the total number of initially falling particles within the screening distance. Since the DEM simulations were conducted with initially 10, 25, 50, 75, 100, and 120 particles, $\lambda_{\mathrm{Dp}}=12.5 \mathrm{~mm}$ was chosen as the screening distance of particles to ensure even at the lowest particle concentration $(n=10)$, at least two particles were located inside the screening distance of any target particle.

Figure 4 includes the effect of electrical particleparticle interactions, as it compares the obtained results from Lagrangian-based approach with formerly developed Eulerian-based approach in the absence of electrical interactions (using Eq. (13)) ${ }^{23}$

$$
\eta=\frac{6 \mathrm{~V} \varepsilon_{0} \phi_{\mathrm{s}}}{\rho_{\mathrm{p}} \mathrm{g}_{1}^{2} \mathrm{D}^{2} \mathrm{~d}_{\mathrm{p}}^{2}}\left[\sqrt{\frac{24 \varepsilon_{0} \phi_{\mathrm{s}}^{2}}{\rho_{\mathrm{p}} \mathrm{d}_{\mathrm{p}}{ }^{2}}+2 \mathrm{~g}_{\mathrm{l}} \mathrm{L}}-2 \frac{\phi_{\mathrm{s}}}{\mathrm{d}_{\mathrm{p}}} \sqrt{\frac{6 \varepsilon_{0}}{\rho_{\mathrm{p}}}}\right]^{2} .
$$

In the absence of electrical particle-particle interaction, providing about $110 \mathrm{~V}$ between ELDC plates is adequate to get $100 \%$ collection efficiency. Apparently from Fig. 4, estimated collection efficiency from the DEM model (dashed line for Lagrangian-based approach with uniform e-field) at this applied voltage is lower than that determined by the analytical model (Eulerian-based approach). Figure 4 indicates for obtaining any collection efficiency greater than about $46 \%$, the Lagrangian-based model is more conservative, asking for higher required voltage and vice versa.

The observed behavior is due to the repelling forces among the like charged particles. After starting a simulation, the 50 particles originally located in the closer side of the centerline with respect to the collecting plate repel the other 50 particles originally located in the further side of the centerline. Consequently, these particles at the closer side accelerate toward the collecting plate faster than what would be

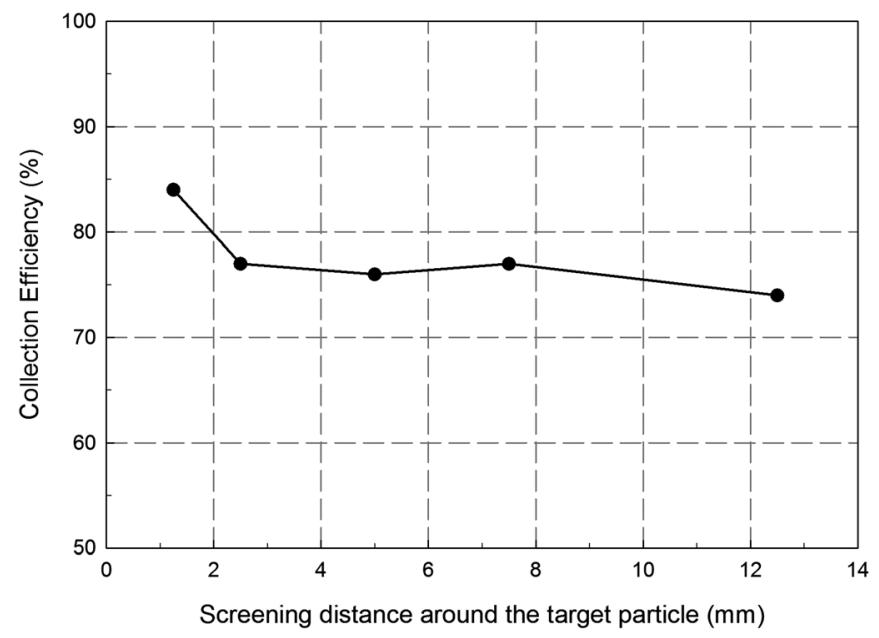

FIG. 3. Sensitivity analysis on electrical screening distance around the particles (one hundred 20- $\mu$ m-sized particles at uniform electrostatic field; $\mathrm{L}=\mathrm{W}=10 \mathrm{~cm}, \mathrm{D}=5 \mathrm{~cm}$, and $\Delta \mathrm{V}=100 \mathrm{~V})$. 


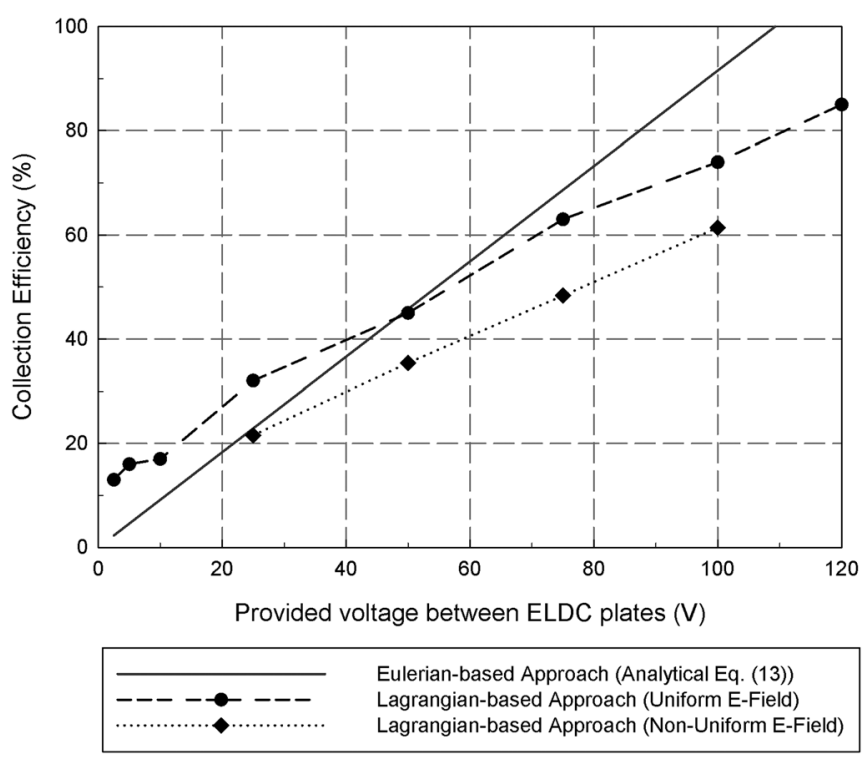

FIG. 4. Collection efficiency as a function of provided voltage via 3 different models: Lagrangian-based approach with a uniform e-field, Lagrangianbased approach with a non-uniform e-field, and Eulerian-based approach. In all 3 models: $\mathrm{d}_{\mathrm{p}}=20 \mu \mathrm{m}, \phi_{\mathrm{s}}=100 \mathrm{~V}, \mathrm{~L}=\mathrm{W}=10 \mathrm{~cm}$, and $\mathrm{D}=5 \mathrm{~cm}$ ).

expected. On the other hand, the other 50 particles at the further side are repelled away from the collecting plate. Later on, the repelling effect on these particles fades away and these particles start to move toward the collecting plate with a small delay. In other words, initially the further side particles sacrifice themselves to expedite collecting process of the other 50 particles. $^{23}$ This is also the reason for observing the intersection between the models close to $50 \%$ collection efficiency.

Using the Eulerian-based model, the expected value for the collection efficiency at $\Delta \mathrm{V}=100 \mathrm{~V}$ is $91 \%$. The result of sensitivity analysis on particle number concentration at this certain applied voltage is shown in Fig. 5. This figure indicates the collection efficiency decreases with an increase in the initial number of falling particles. Since the initial distances among the particles at higher number concentration is shorter, the observed effect of electrical particle-particle interactions at higher particle concentration is greater.

Figure 4 displays the extra electrostatic field required due to the electrical particle-particle interactions. On this basis, about $29 \%$ more electrostatic field should be provided to ensure $100 \%$ collection efficiency of one hundred 20- $\mu$ m-sized lunar dust particles. At $\Delta \mathrm{V}=120 \mathrm{~V}$, the total required electrostatic field would be $2760 \mathrm{~V} / \mathrm{m}$ (see dashed line in Fig. 4). Furthermore, the lowest value of collection efficiency is not zero which is distinct at low provided voltages $(\Delta \mathrm{V}=2.5,5$, and $10 \mathrm{~V})$. Therefore, it can be concluded that even without providing an electrostatic field, electrical particle-particle interactions help to collect a small fraction of particles.

\section{B. Effect of non-uniform charge distribution on ELDC plates}

Before starting the DEM simulations on a non-uniform electrostatic field, capacitance of the ELDC with the above

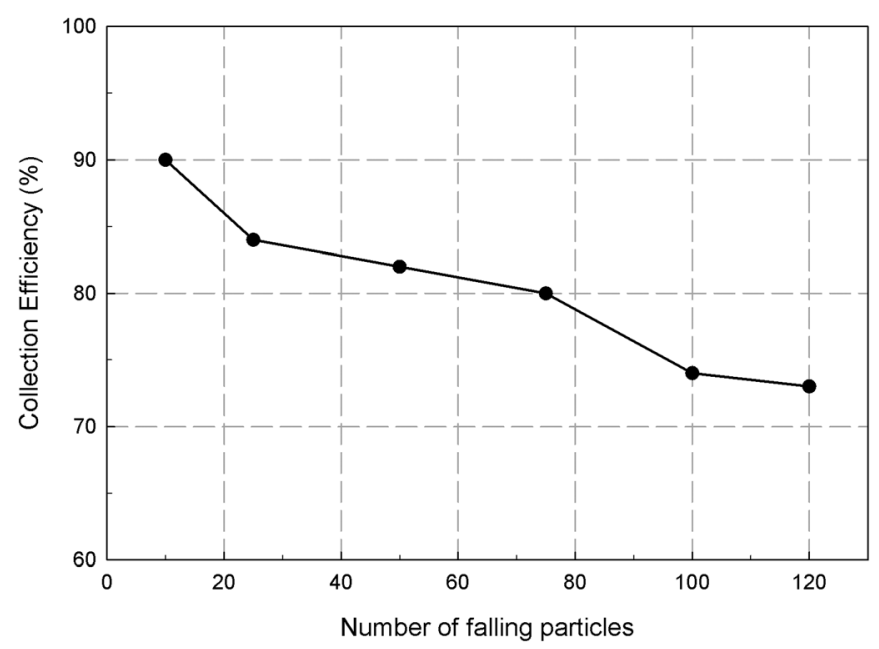

FIG. 5. Effect of particle number concentration on collection efficiency of the ELDC $(\mathrm{dp}=20 \mu \mathrm{m}, \phi \mathrm{s}=100 \mathrm{~V}, \Delta \mathrm{V}=100 \mathrm{~V}, \mathrm{~L}=\mathrm{W}=10 \mathrm{~cm}$, and $\mathrm{D}=5 \mathrm{~cm})$.

mentioned geometry at different numbers of subsections was calculated. Obviously, a higher number of subsections results in more accurate results for the capacitance and charge distribution of subsections. The relationship between the ELDC capacitance and the number of subsections on the ELDC plates was investigated by numerical computations as shown in Fig. 6. This figure indicates the capacitance of the above mentioned ELDC geometry has an asymptotic trend approaching $\mathrm{C}=3.9 \mathrm{pF}$, and for any number greater than 10 (number of sub-sections on side of the plate), the difference is less than $2 \%$. Hence, 100 subsections were opted for discretizing the ELDC plate. The results of non-uniform charge distribution on ELDC plates have been tabulated as in Fig. 7. Figure 4 also displays the obtained DEM results (dotted line) comparing with the previous simpler models.

From the dotted plot in Fig. 4, apparently the collection efficiency reduces due to fringe effect. Surface charges tend to leave the plate center and approach the edges. Hence, wherever particles start to fall, they experience 3 different stages of electrostatic field. Initially the electrostatic field is

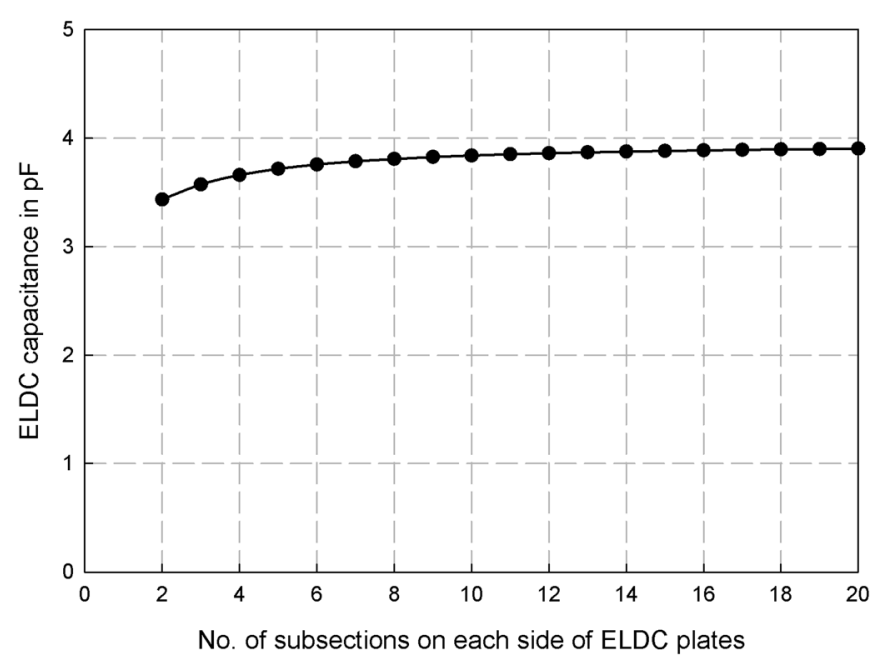

FIG. 6. The ELDC capacitance at different number of subsections $(\mathrm{L}=\mathrm{W}=10 \mathrm{~cm}$ and $\mathrm{D}=5 \mathrm{~cm}$ at $\Delta \mathrm{V}=100 \mathrm{~V})$. 

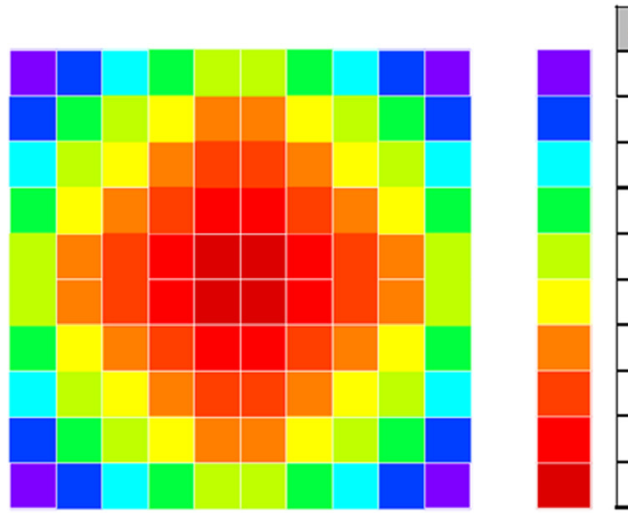

\begin{tabular}{|c|c|c|c|}
\hline$\Delta \mathrm{V}=100 \mathrm{~V}$ & $\Delta \mathrm{V}=75 \mathrm{~V}$ & $\Delta \mathrm{V}=50 \mathrm{~V}$ & $\Delta \mathrm{V}=25 \mathrm{~V}$ \\
\hline 11.689 & 8.767 & 5.844 & 2.922 \\
\hline 9.337 & 7.002 & 4.668 & 2.334 \\
\hline 7.961 & 5.970 & 3.980 & 1.990 \\
\hline 6.984 & 5.238 & 3.492 & 1.746 \\
\hline 6.227 & 4.670 & 3.113 & 1.557 \\
\hline 5.608 & 4.206 & 2.804 & 1.402 \\
\hline 5.085 & 3.814 & 2.543 & 1.271 \\
\hline 4.632 & 3.474 & 2.316 & 1.158 \\
\hline 4.232 & 3.174 & 2.116 & 1.058 \\
\hline 3.875 & 2.906 & 1.937 & 0.969 \\
\hline & & &
\end{tabular}

FIG. 7. Distribution of surface charge on ELDC plates at 4 different provided voltages $(\mathrm{pC})$.

relatively strong. So, in spite of the repelling forces from the $50 \%$ particles which are closer to the collecting plate on the $50 \%$ particles which are further away from the centerline, the stronger electrostatic field dominates the initial repelling actions and more particles move toward the collecting plates. Thus, initially all the particles approach the collecting plate faster than the case of a uniform e-field, and a fraction of closest particles would be collected quicker than the case of a uniform e-field.

When most particles have moved toward the collecting plate, the distances between the particles become shorter, and the electrical repelling interactions increase. The collecting electrostatic forces from point charges on the center of subsections and repelling Coulomb forces from charged particles on any assumed particle are both proportional to particle distance squared, but the relatively stronger electrostatic field shortens the distances among approaching particles. Hence, for any particle there is a balance point inside the ELDC where these two sets of forces equalize and thereafter, repelling Coulomb forces dominate the particle motion. If this balance point for a particle is close enough to the collecting plate, the particles would be collected due to lack of adequate number of repelling particles between the target particle and the collecting plate. On the contrary, if the balance point is relatively far away from the collecting plate, there would be enough number of like charged particles between the target particle and the plate to repel the target particle away from the collecting plate.

The above mentioned balance point for the majority of the particles occurs inside the central region of the ELDC possessing a weaker electrostatic field. When the moved away particles are in the bottom section of the ELDC, the attractive electrostatic field which is again symmetrically strong may not be adequate to collect them before they pass the ELDC. This expresses the reason for observing the intersection of Eulerian-based model (solid line) and Lagrangian-based model (dotted line) at $\eta=22.2 \%$. The ELDC collection efficiency with a non-uniform e-field strongly varies with location, and the particle collection ends up with a lower collection efficiency compared to a uniform electrostatic field. However, the observed decrease in collection efficiency due to the fringe effect is not severe and does not influence the feasibility of the ELDC. According to Table I, required voltage for $100 \%$ collection efficiency with non-uniform electrostatic field is $172.7 \mathrm{~V}$ which is equivalent to less than $3.5 \mathrm{kV} / \mathrm{m}$ e-field strength for the opted ELDC geometry. Moreover, the electrostatic field close to the entrance of the ELDC bends out of the ELDC, and this phenomenon reduces some lunar dust entering the ELDC.

\section{Effect of ELDC dimensions and particle concentrations}

The sensitivity of 3D DEM simulations which deals with point charges at the center of subsections on ELDC plates is different from the derived 2D equation by Eulerian model. ${ }^{17}$ The final set of simulations was conducted to study the influence of ELDC dimensions on the collection efficiency. For this purpose, a half-size ELDC $(\mathrm{L}=\mathrm{W}=5 \mathrm{~cm}, \mathrm{D}=2.5 \mathrm{~cm})$ was considered and all the other conditions were kept the same as the previous set of simulations. The obtained results from DEM modeling are displayed as dashed line and dotted line in Figure 8. Results obtained from the 2D Eulerian-based model using Eq. (13) are also plotted as solid lines in Fig. 8.

The obtained collection efficiencies from the Lagrangian-based model for the half-size ELDC are greater than those obtained from larger ELDC and those obtained from the Eulerian model. This is due to the 3D nature of the ELDC plates in DEM modeling as halving the dimensions decreases the distances between any falling particles and point charges at the center of the subsections on ELDC plates.

Although collection efficiency is not proportional to L/D in the Eulerian-based model, its predicted collection efficiencies for both ELDCs are very similar. The electrostatic force between any individual particle and point charges on the collecting plate increases proportionally to particle distance

TABLE I. Collection efficiency as a function of provided voltage for three applied models.

\begin{tabular}{lccc}
\hline \hline Model & Function & $\mathrm{R}^{2}$ & $\begin{array}{c}\text { Required voltage } \\
\text { at } \eta=100 \% \\
\text { in volts }\end{array}$ \\
\hline $\begin{array}{l}\text { Eulerian-based model } \\
\text { Lagrangian-based } \\
\text { model (uniform E-field) }\end{array}$ & $\eta=0.915 \mathrm{~V}$ & 1 & 109 \\
$\begin{array}{l}\text { Lagrangian-based model } \\
\text { (non-uniform E-field) }\end{array}$ & $\eta=0.5295 \mathrm{~V}+8.57$ & 0.999 & 173 \\
\hline \hline
\end{tabular}



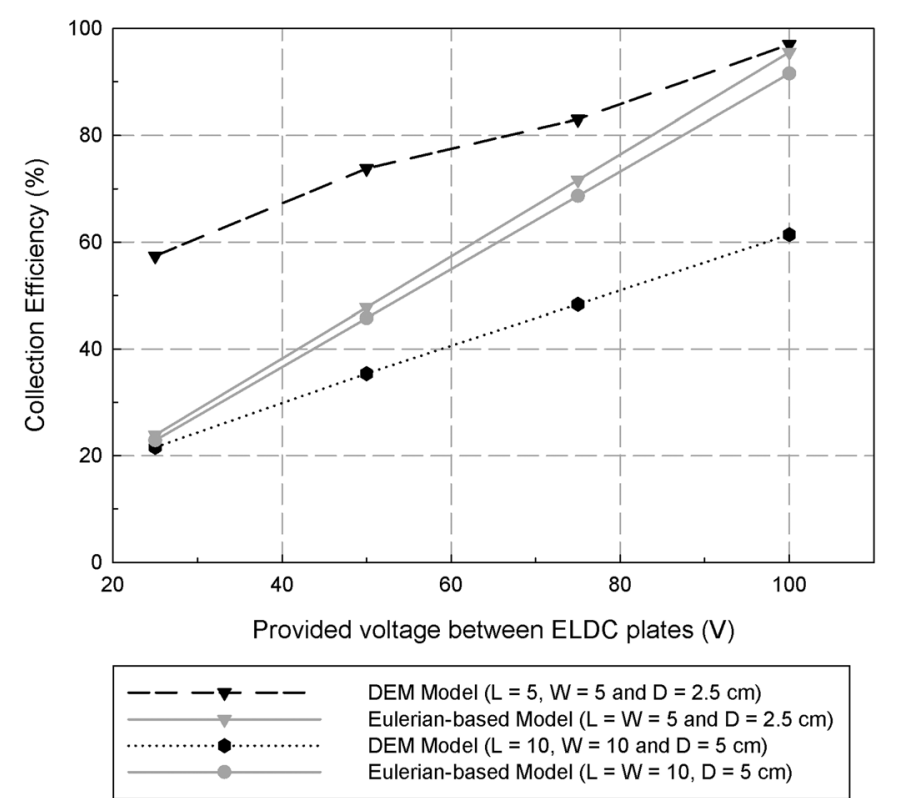

FIG. 8. DEM results for two different sizes of the ELDC and comparison with Eulerian-based model.

squared. Since the 2D Eulerian-based model only exerts uniform electrostatic field on the particles in a single plane and the ELDC width and point charges on ELDC plates are both neglected, the Eulerian model gives similar collection efficiencies as long as $\mathrm{L} / \mathrm{D}$ ratio is constant.

Since charged particles are denser at the ELDC entrance, the effect of electrical particle-particle interactions in particle collection is enhanced even at very low electrostatic field strength. This is why obtained results of DEM modeling for both ELDC sizes in Fig. 8 have similar slopes but the yinterception of a half-size ELDC is significantly greater than the normal-size ELDC. This means a higher concentration of falling lunar particles improves ELDC collection efficiency at relatively lower voltages.

\section{CONCLUSIONS}

The effectiveness of an ELDC for protecting solar panel surfaces from the falling lunar particles in the lunar atmosphere was evaluated by the discrete element method. First, the electrical particle-particle interaction in the uniform electrostatic field was investigated, and the results demonstrated that achieving $100 \%$ collection efficiency for $20-\mu \mathrm{m}$-sized lunar particles required a $29 \%$ higher electrostatic field $(\Delta \mathrm{V}=143 \mathrm{~V}$ for ELDC dimensions of $\mathrm{L}=\mathrm{W}=10 \mathrm{~cm}$ and $\mathrm{D}=5 \mathrm{~cm}$ ) than without electrical particle-particle interaction. This was due to repelling effects of charged particles that caused some particles to move relatively further away from the collection plates. Then, the effect of non-uniformity of the electrostatic field on particle collection was studied by defining 100 subsections on the ELDC plates carrying different amounts of surface charges. The results concluded that non-uniformity of the electrostatic field also decreases the collection efficiency since subsections closer to the edges possess more charges than those closer to the center $(\Delta \mathrm{V}=173 \mathrm{~V})$. However, all the simulations were conducted for the most conservative case scenario and providing less than $3.5 \mathrm{kV} / \mathrm{m}$ electric field was more than enough for collecting this size of lunar particles. Maintaining the electric field at this upper limit is not difficult and does not cause electrical breakdown due to the hard vacuum condition.

The results of the DEM modeling on a half-size ELDC with a non-uniform e-field indicated higher collection efficiencies compared to the Eulerian-based model. This observation was true for the entire range of applied voltages, as the 3D Lagrangian-based model considers ELDC width and includes all point charges on the ELDC plates in Coulomb force calculations. This confirms the conservative nature of Lagrangian-based modeling at $100 \%$ collection efficiency compared to the Eulerian-based model is not generally assured. Depending on the ELDC dimensions and particle concentrations, DEM modeling with less simplifying assumptions may indicate higher collection efficiency. The higher the concentration of incoming lunar dust, the higher the collection efficiency of the ELDC at lower provided e-field. This means time-consuming DEM simulations can be avoided by predicting a more conservative value using the Eulerian-based model.

The obtained results demonstrate the adequacy of the proposed electrostatic lunar dust collection in protection of photovoltaic cells, lenses, and thermal radiators. The possibility of making the ELDC plates from indium tin oxide (ITO) or indium zinc oxide (IZO) material provides transparency along with high conductivity. This means the sunlight blockage does not occur, and performance of the protected solar cells is not deteriorated. While the collection efficiency of the ELDC has been evaluated particularly for collecting charged lunar particles, the obtained results can be extended to any particulate controlling technology in high-vacuum environments utilizing an applied electrostatic field. This study confirms high efficiency of practical electrostatically charged dust collector systems in any high-vacuum environment.

\section{ACKNOWLEDGEMENTS}

This work is supported by Space Research Initiative by the State of Florida (Grant No. 20040028). The authors thank Dr. David R. Curry from DEM-Solutions, Inc., Mr. Nima Gharib from McGill University and Mr. Brian Damit from University of Florida for their help and support in DEM simulations. We also would like to extend our special thanks to Dr. Robert M. Reedy for his editorial comments. The authors are grateful to Particle Engineering Research Center (PERC) at University of Florida for providing EDEM access.

${ }^{1}$ C. I. Calle, J. L. McFall, C. R. Buhler, S. J. Snyder, E. E. Arens, A. Chen, M. L. Ritz, J. S. Clements, C. R. Fortier, and S. Trigwell, in Proc. ESA Annual Meeting on Electrostatics, Minneapolis, MN, 2008, Paper O1.

${ }^{2}$ T. J. Stubbs, R. R. Vondrak, and W. M. Farrell, Adv. Space Res. 37, 59 (2006).

${ }^{3}$ M. M. Abbas, D. Tankosic, P. D. Craven, J. F. Spann, A. LeClair, and E. A. West, Planet. Space Sci. 55, 953 (2007).

${ }^{4}$ N. O. Renno and J. F. Kok, Space Sci. Rev. 137, 419 (2008).

${ }^{5}$ J. Wang, X. He, and Y. Cao, IEEE Trans. Plasma Sci. 36, 2459 (2008).

${ }^{6}$ Z. Sternovsky, S. Robertson, A. Sickafoose, J. Colwell, and M. Horanyi, J. Geophys. Res. 107(E11), 5105, doi:10.1029/2002JE001897 (2002).

${ }^{7}$ L. Belden, K. Cowan, H. Kleespies, R. Ratliff, O. Shah, and K. Shelburne, NASA-CR-190014, NASW-4435 (1991). 
${ }^{8}$ M. Horanyi, Annu. Rev. Astron. Astrophys. 34, 383 (1996).

${ }^{9}$ E. Walbridge, J. Geophys. Res. 78, 3668, doi:10.1029/JA078i019p03668 (1973).

${ }^{10}$ A. Dove, S. Dickson, R. Z. Sternovsky, X. Wang, and M. Horanyi, in 41st Lunar and Planetary Sci. Conf. (Lunar and Planetary Institute, 2010), p. 2406.

${ }^{11}$ Y. J. Pendleton, S. P. Worden, M. Bicay, and J. L. Heldmann, The return to the moon (Cambridge University Press, 2008).

${ }^{12}$ J. E. Colwell, S. R. Robertson, M. Horanyi, X. Wang, A. Poppe, and P. Wheeler, J. Aerosp. Eng. 22, 1 (2009).

${ }^{13}$ V. Pines, M. Zlatkowski, and A. Chait, Planet. Space Sci. 59, 1795 (2011).

${ }^{14}$ Z. Sternovsky, P. Chamberlin, M. Horanyi, S. Robertson, and X. Wang, J. Geophys. Res. 113, A10104, doi:10.1029/2008JA013487 (2008).

${ }^{15}$ O. R. Walton, Grainflow Dynamics, Inc., NASA/CR-2007-214685 (2007).

${ }^{16}$ A. S. Biris, D. Saini, P. K. Srirama, M. K. Mazumder, R. A. Sims, C. I. Calle, and C. R. Buhler, IEEE IAS 2004, 1283.

${ }^{17}$ N. Afshar-Mohajer, B. Damit, C.-Y. Wu, and N. Sorloaica-Hickman, Adv. Space Res. 48, 933 (2011).

${ }^{18}$ R. P. Lin, D. L. Mitchell, D. W. Curtis, K. A. Abderson, C. W. Carlson, J. McFadden, M. H. Acuna, L. L. Hood, and A. Binder, Science 281, 1480 (1998).

${ }^{19}$ A. A. Sickafoose, J. E. Colwell, M. Horanyi, and S. Robertson, Phys. Rev. Lett. 84, 6034 (2000).
${ }^{20}$ C. K. Goertz, Rev. Geophys. 27, 271, doi:10.1029/RG027i002p00271 (1989).

${ }^{21}$ G. H. Heiken, D. T. Vaniman, and B. M. French, Lunar Sourcebook: A User's Guide to the Moon (Cambridge University Press, 1991).

${ }^{22}$ L. A. Taylor, H. H. Schmitt, W. D. Carrier, and M. Nakagawa, in AIAA, 1st Space Exp. Conf.: Continuing the Voyage of Discovery, Orlando, FL, 2005 .

${ }^{23}$ N. Afshar-Mohajer, B. Damit, C.-Y. Wu, and N. Sorloaica-Hickman, in 41st International Conference of Environmental Systems Proceedings, 2011, Portland, OR, AIAA-2011-5201.

${ }^{24}$ M. D. Hogue, C. I. Calle, P. S. Weitzman, and D. R. Curry, J. Electrostat. 66, 32 (2008)

${ }^{25}$ A. Di Renzo and F. P. Di Maio, Chem. Eng. Sci. 59, 525 (2004).

${ }^{26}$ DEM Solutions, Inc., EDEM User Guideline (DEM Solutions, Edinburgh, UK, 2010).

${ }^{27}$ D. J. Loftus, E. M. Tranfield, J. C. Rask, and C. McCrossin, J. of Earth, Moon and Planets 107, 1 (2010).

${ }^{28}$ H. Nishiyama and M. Nakamura, IEEE Trans. Compon., Packag., Manuf. Technol., Part A 17, 477 (1994).

${ }^{29}$ S. Catalan-Izquierdo, J.-M. Bueno-Barrachina, C.-S. Canas-Penuelas, and F. Cavalle-Sese, in Int. Conf. Renewable Energies Power Quality, Valencia, Spain, 2009.

${ }^{30}$ D. K. Reitan and T. J. Higgins, J. Appl. Phys. 22, 223 (1951).

${ }^{31}$ D. K. Reitan, J. Appl. Phys. 30, 172 (1959). 